\title{
The small-molecule compound AC-73 targeting CD147 inhibits leukemic cell proliferation, induces autophagy and increases the chemotherapeutic sensitivity of acute myeloid leukemia cells
}

\section{Isabella Spinello, ${ }^{1}$ Ernestina Saulle, ${ }^{1}$ Maria Teresa Quaranta, ${ }^{1}$ Luca Pasquini, ${ }^{2}$ Elvira Pelosi, ${ }^{3}$ Germana Castelli, ${ }^{3}$ Tiziana Ottone, ${ }^{4}$ Maria Teresa Voso, ${ }^{4}$ Ugo Testa ${ }^{3}$ and Catherine Labbaye ${ }^{1}$}

${ }^{1}$ National Center for Drug Research and Evaluation, Istituto Superiore di Sanità, Rome; ${ }^{2}$ Core Facilities, Istituto Superiore di Sanità; ${ }^{3}$ Department of Oncology and Molecular Medicine, Istituto Superiore di Sanità and ${ }^{4}$ Department of Biomedicine and Prevention, University of Rome "Tor Vergata”, Rome, Italy

\section{ABSTRACT}

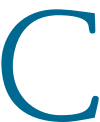

D147 is a transmembrane glycoprotein with multiple functions in human healthy tissues and diseases, in particular in cancer. Overexpression of CD147 correlates with biological functions that promote tumor progression and confers resistance to chemotherapeutic drugs. In contrast to solid tumors, the role of CD147 has not been extensively studied in leukemia. Understanding whether CD147 represents a new hematologic target and whether its inhibitor AC-73 may be used in leukemia therapy may reveal an alternative treatment strategy in patients with acute myeloid leukemia (AML). We analyzed CD147 expression and function in hematopoietic progenitor cells from normal cord blood, in several leukemic cell lines and in primary leukemic blasts obtained from patients with AML. We investigated the effects of AC-73, used alone or in combination with arabinosylcytosine (Ara-C) and arsenic trioxide (ATO), on leukemic cell proliferation. We demonstrated that CD147 overexpression promotes leukemic cell proliferation. We showed that AC-73 exhibits a potent growth inhibitory activity in leukemic cells, by inhibiting the ERK/STAT3 activation pathway and activating autophagy. We demonstrated that AC-73 exerts an anti-proliferative effect additive to chemotherapy by enhancing leukemic cell sensitivity to Ara-C-induced cytotoxicity or to ATO-induced autophagy. We also reported CD147 expression in the fraction of leukemic blasts expressing CD371, a marker of leukemic stem cells. Altogether, our study indicates CD147 as a novel potential target in the treatment of AML and AC-73 as an anti-proliferative drug and an inducer of autophagy in leukemic cells to use in combination with chemotherapeutic agents.

\section{Introduction}

Targeted therapy for acute myeloid leukemia (AML) represents an ongoing challenge and in this context, cluster of differentiation 147 (CD147) represents an attractive target for therapeutic intervention in AML and in other hematologic neoplasms..$^{1-3} \mathrm{CD} 147$, also known as basigin or extracellular matrix metalloproteinase inducer (EMMPRIN), is a type-I transmembrane glycoprotein that belongs to the immunoglobulin superfamily. Among the numerous studies that have documented the significance of CD147 in various physiological processes, the best characterized function of CD147 is related to its role in tumor metastasis, angiogenesis and chemoresistance processes..$^{3-6}$ Overexpression of CD147 correlates with a number of biological functions that promote tumor progression (e.g. cellular proliferation, angiogenesis, matrix metalloproteinase production) and confers resistance to chemotherapeutic drugs such as adriamycin, ${ }^{7,8}$ cisplatin. ${ }^{9} \mathrm{CD} 147$ mediates molecu-
Ferrata Storti Foundation

Haematologica 2019

Volume 104(5):973-985

\section{Correspondence:}

CATHERINE LABBAYE

catherine.labbaye@iss.it

Received: June 11, 2018.

Accepted: November 15, 2018.

Pre-published: November 22, 2018.

doi:10.3324/haematol.2018.199661

Check the online version for the most updated information on this article, online supplements, and information on authorship \& disclosures: www.haematologica.org/content/104/5/973

\section{(C)2019 Ferrata Storti Foundation}

Material published in Haematologica is covered by copyright. All rights are reserved to the Ferrata Storti Foundation. Use of published material is allowed under the following terms and conditions:

https://creativecommons.org/licenses/by-nc/4.0/legalcode. Copies of published material are allowed for personal or internal use. Sharing published material for non-commercial purposes is subject to the following conditions:

https://creativecommons.org/licenses/by-nc/4.0/legalcode, sect. 3. Reproducing and sharing published material for commercial purposes is not allowed without permission in writing from the publisher. 
lar events by interacting with various binding partners, such as tumor- and inflammation-associated molecules including integrins, monocarboxylate transporters (MCTs), cyclophilins, caveolin-1, and E-selectin, explaining its significant role in the pathogenesis of several diseases. ${ }^{3-6,10} \mathrm{CD} 147$ overexpression and more recently its coexpression with MCTs $^{11,12}$ are regarded as unfavorable prognostic factors in cancers associated with hypoxia, a common feature of solid tumors, but also a major component of the bone marrow (BM) microenvironment, crucial in leukemia progression. ${ }^{13,14}$ However, in contrast to solid tumors, the function of CD147 remains poorly defined in leukemia.

Recent studies have shown growing interest in the CD147 molecule in $\mathrm{AML}^{15,16}$ and in some hematologic neoplasia, in particular in multiple myeloma (MM), where CD147 expression levels have a prognostic value and are required for the proliferation of MM cells. ${ }^{17-19}$ Moreover, CD147 is over-expressed in erythroid cells of myelodysplastic syndrome (MDS) with $5 q$ deletion. ${ }^{18}$ Here, we show that CD147 is expressed in normal CD34 hematopoietic progenitor cells (HPCs) and down-regulated during monocytic and granulocytic differentiation of HPCs. We then show that CD147 is over-expressed in blasts pertaining to different subtypes of AML and promotes leukemic cell proliferation. Interestingly, we report that CD147 is expressed at the level of CD $34^{+} \mathrm{CD} 371^{+}$ AML cells, previously described for their leukemia-initiating properties. $^{20}$

Recently, the small-molecule AC-73 has been proposed as a specific inhibitor for CD147. ${ }^{21}$ First, we checked that the response to AC-73 treatment is not involved in an offtarget mechanism in leukemic cells. Then, we analyzed the effects of CD147 inhibition by AC-73 in AML cell lines and in primary leukemic blasts. We found that AC73 inhibits leukemic cell proliferation by suppressing the ERK/STAT3 activation pathway, known to play a role in AML cell proliferation and survival, ${ }^{22}$ but also by activating autophagy, an essential phenomenon for hematopoietic stem cell (HSC) maintenance, resistance to stress, survival and differentiation, the machinery of which might be disrupted in AMLs. ${ }^{23-25}$ Next, we analyzed whether AC-73 enhanced the sensitivity of leukemic cells to conventional chemotherapeutic agents. We used arabinosylcytosine (Ara-C), one of the most active cytotoxic agents in myeloid leukemia, and arsenic trioxide (ATO), an active anti-proliferative agent used in the treatment of patients with acute promyelocytic leukemia (APL) (AML-M3) ${ }^{1,2,26}$ [although with low efficacy in AML lacking the $t(15 ; 17)$ translocation], and also an inducer of autophagy. ${ }^{25,27} \mathrm{We}$ found that AC-73 used in vitro in combination with Ara-C or ATO, increases the effects of these agents.

Altogether, our data suggest that CD147 plays a key role in leukemic cell proliferation and represents a potential therapeutic target in AML patients, and that AC-73 is a new promising inhibitor that could be used in combination with conventional chemotherapeutic agents as a novel treatment strategy in AML.

\section{Methods}

\section{Cell cultures}

Human cord blood (CB) was obtained from healthy donors after informed consent. Leukemic blasts were isolated from BM obtained from patients with newly diagnosed AML, using FicollHypaque density gradient. Informed consent was obtained from patients in accordance with the Declaration of Helsinki. This study was approved by the local ethical committees of the Istituto Superiore di Sanita and the University of Tor Vergata, Rome, Italy.

Cord blood CD34 ${ }^{+}$HPC purification, unilineage monocytic (Mo) and granulocytic (G) differentiation and morphological analyses were performed, ${ }^{28,29}$ as described in the Online Supplementary Methods

Human primary AML blasts were maintained in culture in Iscove medium supplemented with 10\% FCS, GM-CSF (10 $\mathrm{ng} / \mathrm{mL}), \mathrm{SCF}(50 \mathrm{ng} / \mathrm{mL}), \mathrm{IL}-3$ (10 ng/mL) (PeproTech Inc., Rocky Hill, NJ, USA), as described. ${ }^{29}$

Human AML cell lines used in our study were: U937 as a model of AML-M5, NB4 and HL-60 as models of AML-M3 and AMLM4, respectively; NB4-R4 as AML-M3 resistant to all-trans retinoic acid (ATRA) treatment; MV4-11 as AML-M2 mutated for FLT3ITD; Kasumi-1 as AML-M2 with the t(8;21) translocation. All cell lines were grown in RPMI medium supplemented with $10 \%$ FCS (Gibco, Carlsbad, CA, USA).

Cell growth, cell cycle profile, viability and apoptosis analysis were performed, as described in the Online Supplementary Methods.

Flow cytometry, western blot and quantitative real-time RTPCR analysis were as described in the Online Supplementary Methods.

Knockdown of CD147 expression by RNA interference, clonogenic assays and colony formation assays were performed, as described $^{30}$ in the Online Supplementary Methods.

Cyto-ID autophagy detection was performed using the CytoID assay (Enzo Life Sciences ENZ-51031-K200) as described in the Online Supplementary Methods.

\section{AC-73 treatment of leukemic cells used alone or in combination with chemotherapeutic agents}

AC-73 (3-\{2-[([1,1'-biphenyl]-4-ylmethyl) amino]-1-hydroxyethyl)phenol) (Specs ID number AN-465/42834501, Specs, Zoetermeer, the Netherlands) was dissolved in 20\% DMSO (Sigma, St. Louis, MO, USA) and diluted in DMEM, with a final DMSO concentration of no more than $0.2 \%$ for all in vitro studies. ${ }^{21}$ In leukemic cell lines, dose-response and time-course analysis were performed using AC-73 at 1.0, 2.5, 5.0 and $10 \mu \mathrm{M}$ from 1 to 4 days of treatment; results were compared with $0.2 \%$ DMSOtreated leukemic cells, indicated as control leukemic cells. AC-73 was added in cultures every 2 days to maintain its activity. Combinations of treatment were performed using AC-73 $(2.5 \mu \mathrm{M})$ alone over 24 hours and then by adding Ara-C $(0.01 ; 0.1$ and 1.0 $\mu \mathrm{M}$ ) or ATO $(0.1 ; 0.01$ and $1.0 \mu \mathrm{M}$ ) for another 1 (for NB4 and NB4-R4 cells) or 2 (for U937, HL-60, MV4-11 and Kasumi-1 cells) days. Cell viability assays were performed to evaluate the effect of AC-73 alone or in combination on cell growth and viability of these cells.

\section{Analysis of The Cancer Genome Atlas data}

Datasets of The Cancer Genome Atlas (TCGA) Research Network 2008, were processed and obtained directly from the public access data portal (http://tcga-data.nci.nih.gov).

\section{Statistical analysis}

Student $t$-test was applied to assess statistical significance of differences between multiple/group of experiments. Data were analyzed using GraphPad Prism software. For univariate survival analysis, Kaplan-Meier plots with a log-rank test were presented using the overall survival data of AML patients from the TGCA. Additional information is provided in the Online Supplementary Appendix. 


\section{Results}

CD147 is directly involved in hematopoietic progenitor cell proliferation

We analyzed CD147 expression at both mRNA and protein level in CD34 $4^{+} \mathrm{HPCs}$ and during Mo and G proliferation and differentiation of these cells. CD147 is well expressed in CD34+ HPCs and its level of expression decreases during Mo and $G$ differentiation and maturation of these cells (Figure 1A-C and Online Supplementary Figure
S1A and B). Western blot analysis shows that CD147 protein is highly glycosylated (HG-CD147 $40-60 \mathrm{kDa}$ ) in CD34 $4^{+}$HPCs and at all stages of Mo and G cell differentiation (Figure 1B), indicating the presence of CD147 protein in a stable and biologically active conformation mainly translocated to plasma membrane, ${ }^{5}$ as also shown by flow cytometry analysis (Figure 1C and Online Supplementary Figure S1B). To analyze the role of CD147 in HPCs, we performed siRNA-mediated CD147 knockdown experiments in CD $34^{+}$HPCs. We transiently trans-
A

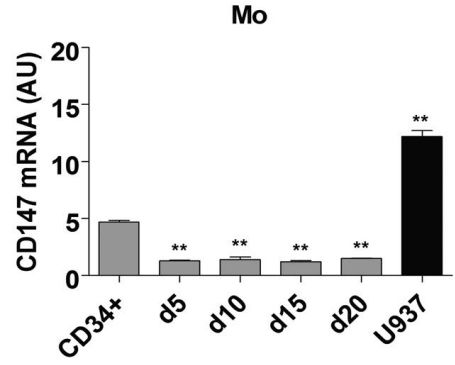

B

Mo

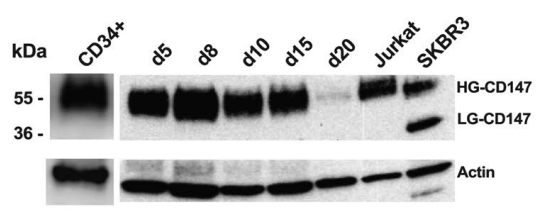

C

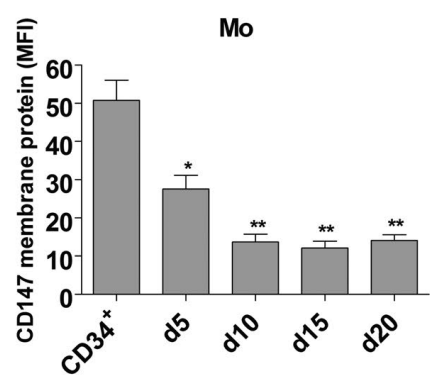

G

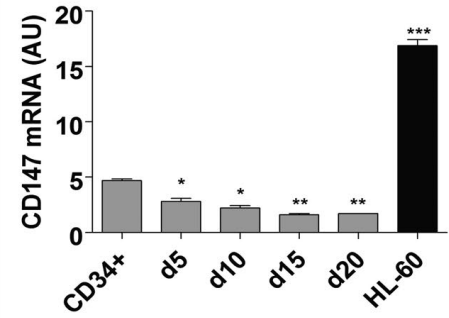

G

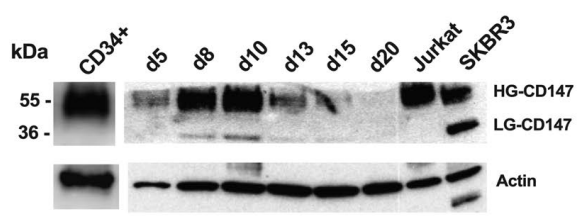

G

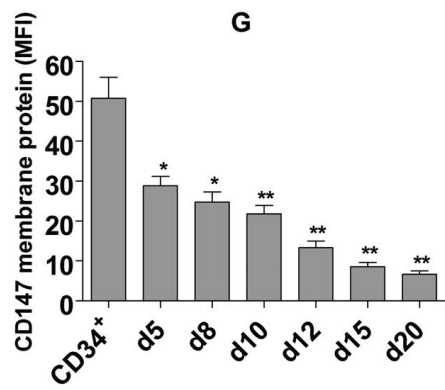

Figure 1. CD147 is down-regulated during monocytic (Mo) and granulocytic (G) differentiation of $\mathrm{CD} 4^{+}$hematopoietic progenitor cells (HPCs) and is involved in HPC proliferation. (A) qRT-PCR analysis of CD147 mRNA expression during selective Mo and $\mathrm{G}$ proliferation and differentiation of $\mathrm{CD} 4^{+} \mathrm{HPCs}$, as compared to U937 and $\mathrm{HL}-60$ leukemic cells. (B) Western blot analysis of CD147 protein expression level in CD34 ${ }^{+}$cells and Mo and $\mathrm{G}$ differentiating HPCs; Jurkat and SKBR3 cells are shown as positive controls of CD147 expression; HG- and LG- are indicated for High- and Low- glycosylated CD147 isoforms; actin is shown as an internal control; molecular weights are indicated (kDa). (C) Flow cytometry analysis of CD147 membrane protein expression during Mo and $G$ differentiation and maturation of HPCs. (D) Flow cytometry analysis of CD147 membrane protein expression in Mo and $\mathrm{G}$ differentiating transfected (CD147-siRNA)-HPCs, as compared to transfected (c-siRNA)-HPCs of control, at day 2 of Mo and G cultures corresponding to day 3 post transfection. (E) Cell growth inhibition of (CD147-siRNA)-HPCs, as compared to (c-siRNA)-HPCs, grown under both $\mathrm{G}$ and Mo liquid culture conditions. (F) Clonogenic assays performed under $\mathrm{G}$ and Mo culture conditions with (CD147siRNA)-HPCs, as compared to (c-siRNA)HPCs. (A, C-F) Mean \pm Standard Error of Mean of three independent experiments is shown. ${ }^{*} P<0.05$; $* * P<0.01$; $\star * * P<0.001$. (B) One representative experiment out of three is shown. AU: arbitrary units; MFI: mean fluorescence intensity.
D

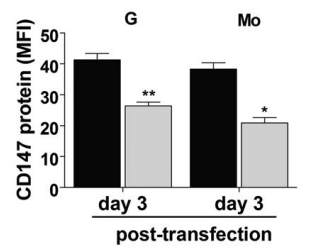

F

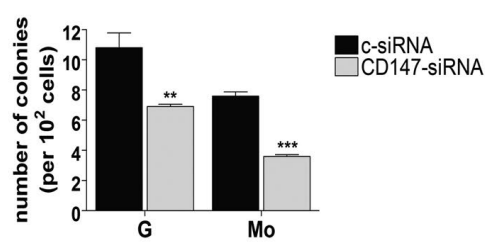

E

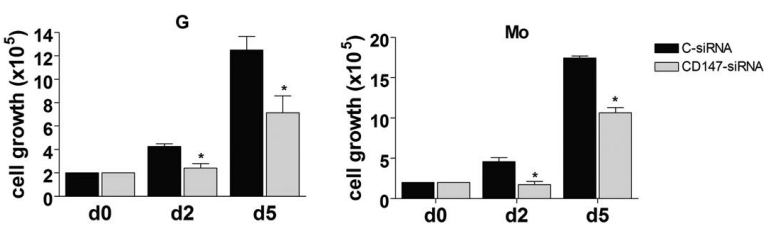


fected HPCs with specific-CD147 interfering small RNAs (CD147-siRNA) or with a non-targeting control siRNA (csiRNA). Silencing of CD147 mRNA decreased CD147 protein levels in transfected (CD147-siRNA)-HPCs, as compared to (c-siRNA)-HPCs of control (Figure 1D); then, transfected-HPCs were grown under culture conditions, to allow their selective $\mathrm{G}$ or Mo proliferation and differentiation. We observed that the decreased expression of CD147 impaired the proliferation of both G and Mo differentiating (CD147-siRNA)-HPCs, as compared to control (c-siRNA)-HPCs (Figure 1E), without any significant modulation of $\mathrm{G}$ or Mo specific antigen expression on these cells (data not shown) and without any significant induction of apoptosis (data not shown). Clonogenic G and Mo progenitor assays performed with transfected-HPCs, showed a significant decrease in the number of CFU-GM and CFU-M colonies obtained from (CD147-siRNA)HPCs, compared with the number of colonies obtained from (c-siRNA)-HPCs (Figure 1F). Altogether, our data indicate that CD147 plays a role in HPC proliferation and clonogenic activity.

\section{Over-expressed in AML, CD147 is down-regulated during differentiation of leukemic cells}

By analyzing CD147 mRNA expression levels in primary leukemic blast cells obtained from 48 patients with different subtypes of AML, ${ }^{29}$ we found that CD147 is over-expressed in all AML subtypes, in particular in the AML-M3 subtype, as compared to normal CD34+ HPCs (Figure 2A, left panel), in line with the data from AML samples generated by the TCGA Research Network (Figure 2A, right panel).

Then, we also examined CD147 expression in several AML cell lines induced to terminal differentiation. All leukemic cell lines analyzed over-expressed the fully glycosylated mature CD147 protein, biologically active (Figure 2B, HG-CD147), like also its mRNA, as compared to normal CD34+ HPCs (Figure 2C). We found that CD147 expression is down-regulated during vitamin D3-induced Mo differentiation of U937 cells and ATRA-induced G differentiation of HL-60 and NB4 cell lines, at both mRNA and protein levels during terminal differentiation (Figure 2D-F and Online Supplementary Figure S1C-H), similarly to $\mathrm{Mo}$ and $\mathrm{G}$ terminal differentiation of normal HPCs (Figure 1A-C). However, CD147 expression is not affected by ATRA treatment of the NB4-R4 cell line (Figure 2G and Online Supplementary Figure S1I and L), indicating that induction of cell differentiation is required for CD147 downregulation.

\section{AC-73 inhibits leukemic cell proliferation by blocking ERK/STAT3 signaling and induces autophagy}

To investigate whether a high level of CD147 can promote cell proliferation, we used AC-73 to inhibit CD147 function in normal and leukemic cells.

First, we examined the effect of AC-73 treatment on G and Mo differentiation of normal HPCs. AC-73 used at 5 $\mu \mathrm{M}$ moderately decreased cell growth without affecting cell cycling or differentiation of HPCs (Figure 3A-C). Morphology analysis of HPCs at different days of culture supported these observations, showing that AC-73-treated cells regularly progress along $\mathrm{G}$ and Mo differentiation and do not show any significant morphology abnormality or delay in differentiation (Figure 3D).

We analyzed the effects of chronic AC-73 administra- tion on leukemic cell growth, apoptosis and viability of AML cell lines. Leukemic cells were treated from 1 to 3 days, with various AC-73 doses $(2.5,5$ and $10 \mu \mathrm{M})$ to determine their sensitivity to AC-73 treatment. We observed a time- and dose-dependent effect of AC-73 on leukemic cell growth, with growth inhibition of all leukemic cell lines treated (Figure 4A and Online Supplementary Figure S2B). We could not detect any effect of AC-73 on cell growth and apoptosis of the Chinese Hamster Ovary (CHO) cells (data not shown) used as a CD147-negative cell line (Online Supplementary Figure S2A, left panel).

NB4 and NB4-R4 cell lines were more sensitive to AC73 treatment than U937 and HL-60 cell lines. Indeed, a higher significant decrease in cell growth (Figure 4A), apoptosis (Figure 4B), and viability (Figure 4C and D) was observed in NB4 and NB4-R4 cells treated with low-dose AC-73 $(2.5 \mu \mathrm{M})$ than in U937 and HL-60 lines (2 days) (Figure 4D). MV4-11, Kasumi-1 cells were the most resistant to AC-73 treatment (Online Supplementary Figure S2BE) when compared to other cell lines (Figure 4A-D). Significant cell growth inhibition and sensitivity of MV411 and Kasumi-1 cells occurred after treatment with high concentration of AC-73 (5 and $10 \mu \mathrm{M}$ ) (Online Supplementary Figure $S 2 B-D$ ) or when cells were treated for longer (3-4 days) (Online Supplementary Figure S2E).

AC-73 treatment had no significant effect on cell cycle distribution in the leukemic cell lines tested as compared to control cells (data not shown), suggesting that AC-73 decreases the cell growth rate but does not inhibit the progression of cells in the cell cycle. Analysis of CD11b, CD14 and CD15 expression levels in leukemic cell lines treated for 3 days with $5 \mu \mathrm{M}$ AC-73 showed no significant effect of AC-73 on leukemic cell differentiation (data not shown). By analyzing the effects of AC-73 $(5 \mu \mathrm{M})$ on clonogenic growth of U937 and NB-4 cells we found that AC73 decreases the in vitro colony formation of both U937 and NB-4 cell lines, indicating an inhibitory effect of AC73 on the clonogenetic capacity of leukemic cells (Online Supplementary Figure S2F).

Altogether, despite the different sensitivities to AC-73 manifested by the different leukemic cell lines, probably related to the specific molecular alteration, such as PML/RARA, FLT3-ITD or RUNX1/RUNX1T1, of these cell lines, AC-73 exhibits potent growth inhibition and cytotoxic activity on leukemic cells only at high doses. Because low doses of AC-73 inhibit leukemic cell proliferation, but do not cause cell death via apoptosis or cell cycle arrest, we investigated the possibility that AC-73 treatment induces autophagy in leukemic cells. First, we assessed the effect of $\mathrm{AC}-73$ on the level of the autophagic indicator LC3 by western blotting in U937 and NB4 cells. Our results showed a dose-dependent effect of $\mathrm{AC}-73$ on the increase of LC3-II/LC3-I ratio in leukemic cells as compared to control (-) cells (Figure 4E), indicating induction of autophagy. ${ }^{31}$ Then we monitored autophagy flux by flow cytometry analysis in live leukemic cells treated for 72 hours (h) by AC-73 compared to control cells. Our data demonstrated that AC-73 induces dose-dependent autophagy (Figure 4F) in CD147-expressing leukemic cells (Figure 4G), but not in CHO cells (Online Supplementary Figure S2A, right panels), again indicating that the biological effects induced by $\mathrm{AC}-73$ require $\mathrm{CD} 147$ expression on target cells. Considering that AC-73 suppresses CD147/ERK1/2/STAT3/MMP-2 pathways in hepatocellu- 
lar carcinoma cells, and that ERK/STAT3 signaling plays a significant role in promoting AML cell proliferation, survival and autophagy, ${ }^{22,23,32,33}$ we also investigated the effect of AC-73 on ERK1/2 and STAT3 activation in leukemic cells. In line with previous studies, ${ }^{31,32}$ western blot analysis showed that ERK and STAT3 are constitutively phosphorylated in all leukemic cell lines [Online Supplementary Figure S3, lanes (-)]. We then found that AC-73 (5 $\mu \mathrm{M}$, for 3 days) notably decreases both pERK and pSTAT3 (S727) levels without affecting the levels of total ERK and STAT3, in leukemic cells (lanes $5 \mu \mathrm{M}$ ) (Online Supplementary Figure S3, lower panels).
Altogether, our data demonstrate that AC-73 inhibits leukemic cell proliferation, in part by suppressing the ERK/STAT3 activation pathway in these cells, in part by activating a non-apoptotic, autophagic form of cell death, which may account for the efficacy of AC-73 in leukemic cells.

\section{AC-73 has an additive anti-proliferative effect on chemotherapeutic treatment of leukemic cells and increases ATO-induced autophagy in leukemic cells}

To evaluate the effect of AC-73 in combination with standard drugs used for AML treatment, AML cell lines
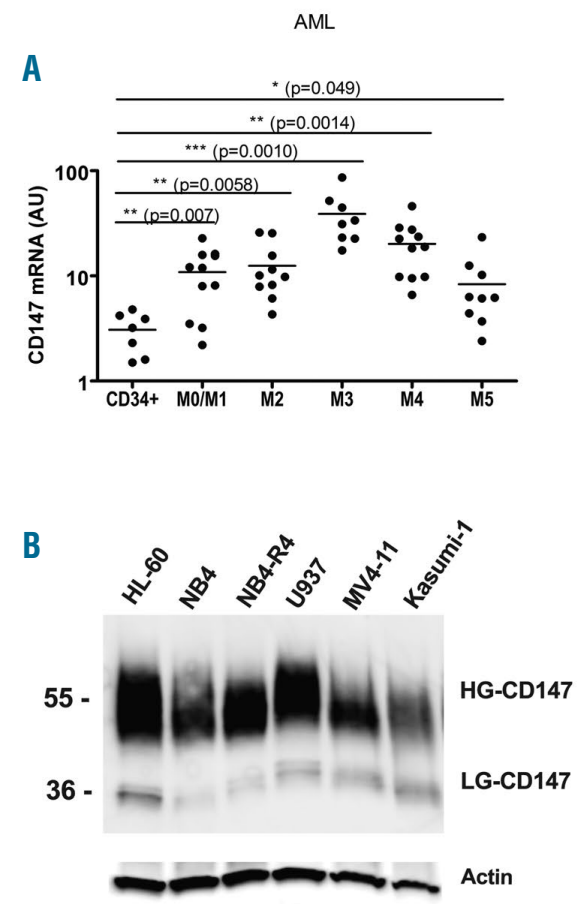

A.U. $1.94 \quad 1.46 \quad 1.69 \quad 1.83 \quad 1.79 \quad 1.93$

D

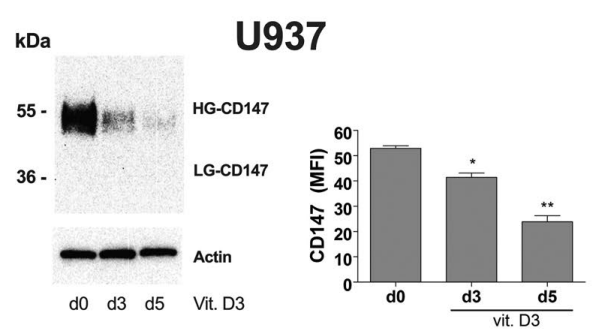

$\mathbf{F}$

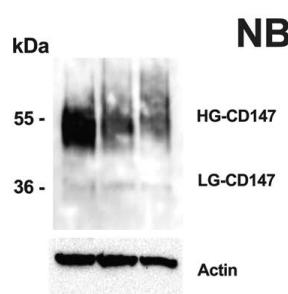

do d1 d2 ATRA
C
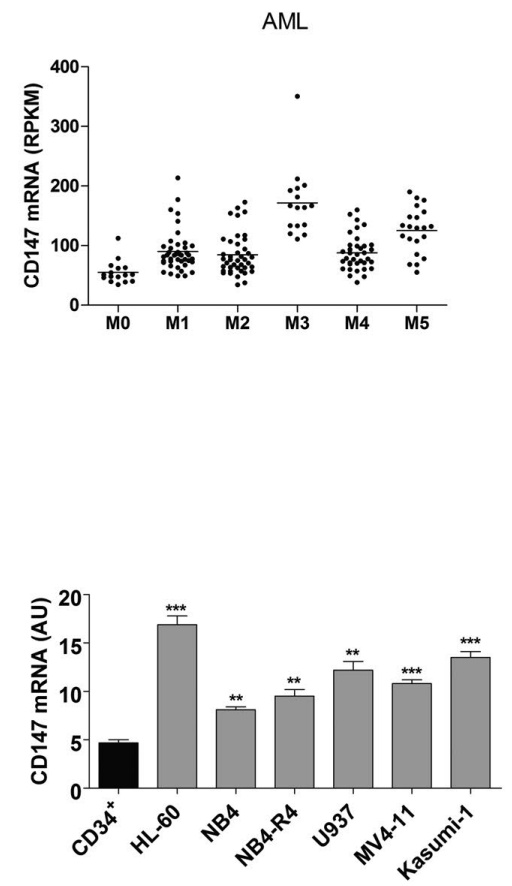

E

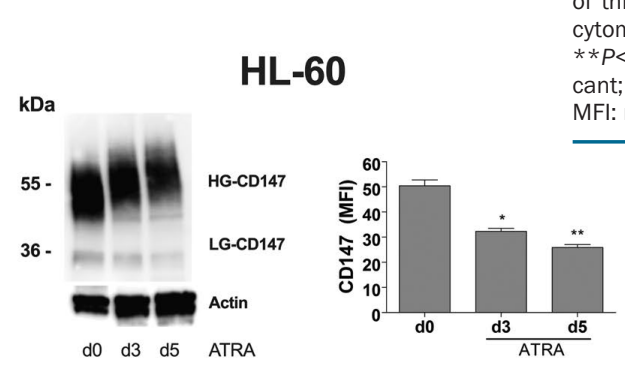

Figure 2. Over-expressed in acute myeloid leukemia (AML), CD147 expression is down-regulated during monocytic and granulocytic differentiation of leukemic cells. (A) qRT-PCR analysis of CD147 mRNA expression in primary leukemic cells of AMLs pertaining from M0 to M5 subtypes of the French-American-British (FAB) classification, as compared to normal $\mathrm{CD}^{+} 4^{+}$ hematopoietic progenitor cells (HPCs) (left panel); CD147 mRNA expression data from AML samples generated by TCGA Research Network (right panel). (B) Western blot analysis of CD147 protein expression level in AML cell lines; densitometry analysis [arbitrary units (AU)] of CD147 protein expression levels compared with actin levels is indicated. (C) qRT-PCR analysis of CD147 mRNA expression in leukemic cell lines, as compared to normal CD34 ${ }^{+}$HPCs. (D-G) Western blot (left panels) and flow cytometry (right panels) analysis of CD147 total and membrane protein expression levels during vitamin D3-induced monocytic differentiation of U937 cell (D), ATRAinduced granulocytic differentiation of $\mathrm{HL}$ 60 cells (E), ATRA-induced differentiation of NB4 cells $(F)$, and in NB4-R4 cells, resistant to ATRA treatment (G). (A and C) Mean \pm Standard Error of Mean (SEM) of three independent experiments is shown. $* P<0.05$; ** $P<0.01$; *** $P<0.001$. (B, D-G, left panels) One representative western blot experiment out of three is shown. High (HG) and Low (LG) glycosylated CD147 isoforms are indicated. Actin is shown as internal control. (D-G, right panels) Mean \pm SEM of three independent experiments by flow cytometry analysis is shown. ${ }^{*} P<0.05$; $* * P<0.01 ; * * * P<0.001$. ns: not significant; RPKM: Reads Per Kilobase Million; MFI: mean fluorescence intensity.

G

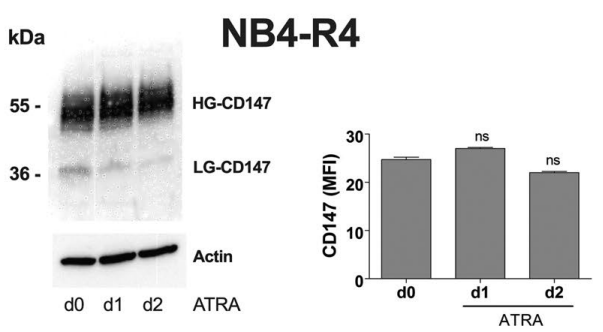


and primary AML blasts were initially treated for $24 \mathrm{~h}$ with a low concentration $(2.5 \mu \mathrm{M})$ of $\mathrm{AC}-73$ and then cotreated for $48 \mathrm{~h}$ with Ara-C or ATO at different concentrations $(0.01-1 \mu \mathrm{M})$. Cell viability assays were performed to assess leukemic cell survival and sensitivity to AC-73 treatment used in combination with Ara-C or ATO, as compared to single AC-73, Ara-C, or ATO treatment (Figure 5A and B and Online Supplementary Figure S4). We observed a significant decrease in leukemic cell viability of all leukemic cell lines after AC-73 treatment in combination with Ara-C or ATO, as compared to AC-73, Ara-C or ATO used alone (Figure $5 \mathrm{~A}$ and $\mathrm{B}$ and Online Supplementary Figure S4). Furthermore, we identified three subgroups of leukemic cells according to their Ara-C drugsensitivity: high-sensitivity lines (U937 and HL-60); intermediate-sensitivity lines (NB4 and NB4-R4); low-sensitivity lines (MV4-11 and Kasumi-1). AC-73 potentiates the sensitivity to Ara-C treatment of all leukemic cells, even those belonging to the low-sensitivity subgroup (Figure $5 \mathrm{~A}$ and B and Online Supplementary Figure S4). The antiproliferative effect of AC-73 enhances sensitivity to ATO treatment of both M3 leukemic cells, such as NB4 and NB4-R4 (Figure 5B and Online Supplementary Figure S4B), and non-M3 leukemic cells, such as U937, HL-60, Kasumi1 and MV4-11 cells (Figure 5A and Online Supplementary Figure $S 4 A, C$ and D).

Then, we showed that when used in combination with Ara-C and ATO, AC-73 treatment inhibits ERK and STAT3 activation in leukemic cells (Figure 5C).

Because ATO is also an inducer of autophagy, ${ }^{25}$ we analyzed the autophagy flux in both $M 3$ and non-M3 leukemic cells treated by ATO or AC-73 used alone or in combination, as compared to control cells. Our data showed that AC-73 and ATO are both inducers of autophagy and the autophagy flux is significantly increased in M3 (NB4) and non-M3 (U937) leukemic cells
G

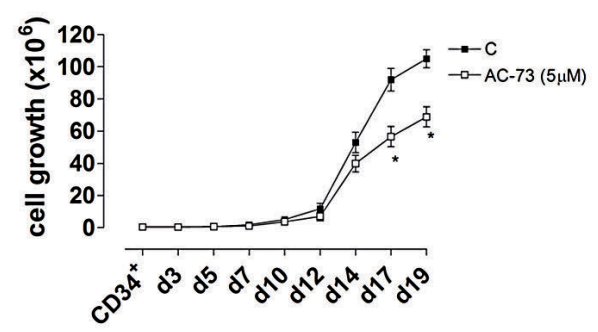

B

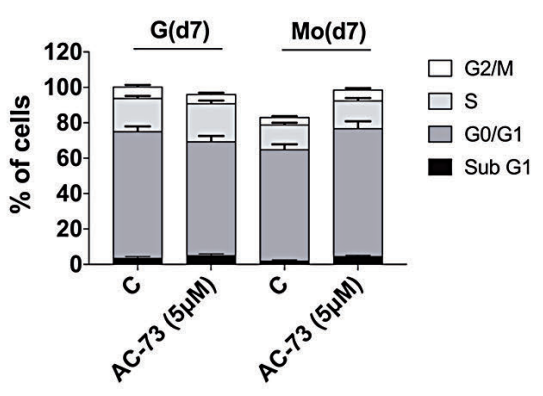

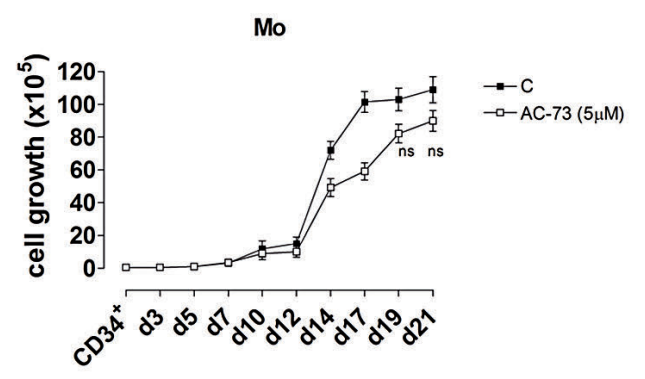

C
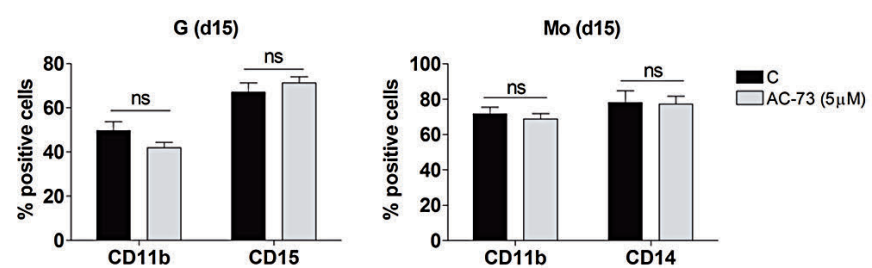

D

G(d7)
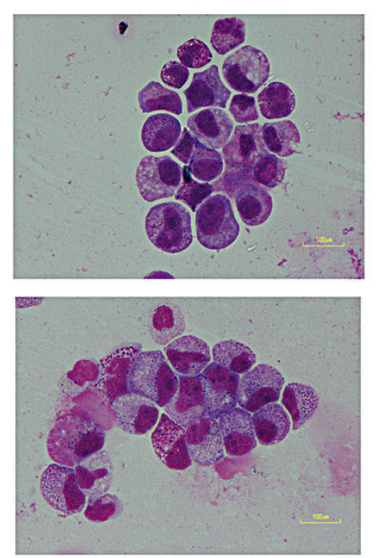

Mo(d7)

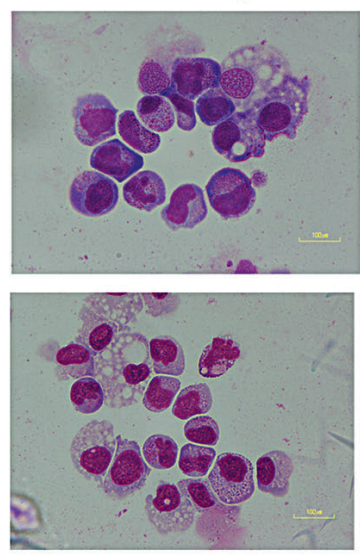

C

AC-73 (5uM)
Figure 3. AC-73 has no significant effect on cel growth, differentiation and cell cycle progression in normal granulocytic (G) and monocytic (Mo) differentiating hematopoietic progenitor cells (HPCs). (A) Cell growth analysis during $\mathrm{G}$ and Mo differentiation of HPCs, in presence of AC-73 used at $5 \mu \mathrm{M}$ and added every 2 days in cultures, as compared to control (C) cells. (B) Cell cycle analysis at day 7 of $\mathrm{G}$ and Mo differentiation of HPCs treated with AC-73 (5 $\mu \mathrm{M})$, as compared to control HPCs. (C) Phenotype analysis performed by analyzing CD11b, CD15 and CD14 expression levels in $\mathrm{G}$ and Mo differentiating HPCs at day 15, in the presence or not (C) of AC-73. (A-C) Mean \pm Standard Error of Mean (SEM) of three independent experiments is shown. $* P<0.05$; ns: not significant. (D) Morphological analysis at day 7 of the differentiation and maturation of $\mathrm{G}$ and Mo differentiating HPCs treated with AC-73, as compared to control (C) HPCs, and stained with May-GrünwaldGiemsa. (D) One representative experiment out of three is shown. 
treated by ATO and AC-73 used in combination, as compared to single agent treatment or control cells (C) (Figure $5 \mathrm{D}$ and $\mathrm{E})$.

Altogether, our data demonstrate that AC-73 is a potent, novel anti-proliferative molecule that enhances the sensitivity of leukemic cells to conventional chemotherapeutic agents, but also increases ATO-induced autophagy in M3 and non-M3 leukemic cells.
AC-73 increases the sensitivity of primary acute myeloid leukemia blasts co-expressing CD147 and CD371 to chemotherapy

We evaluated the effects of AC-73 used alone and in combination with Ara-C or ATO on primary AML blasts. First, we analyzed CD147 protein expression in samples obtained from different subtypes of AML and we controlled the expression of CD34, CD38 and CD371 mark-

A
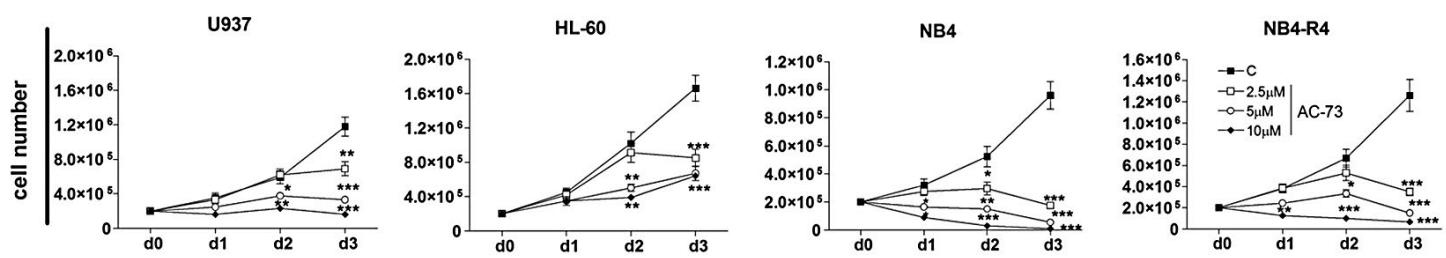

B
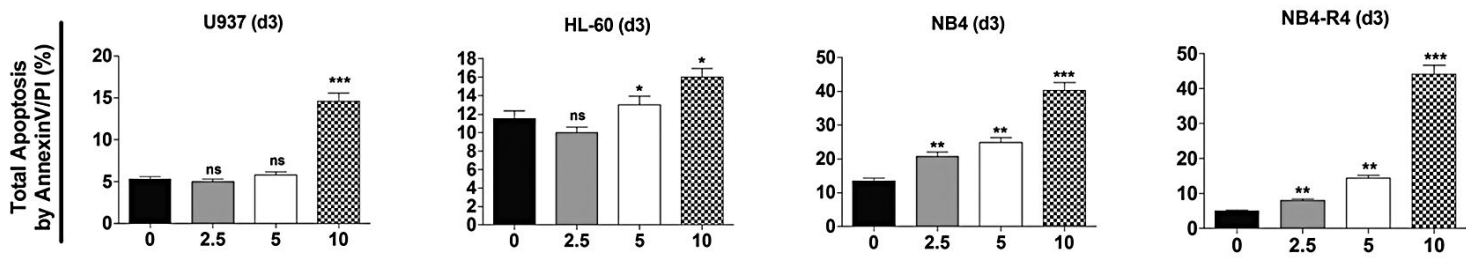

AC-73 $(\mu \mathrm{M})$

C

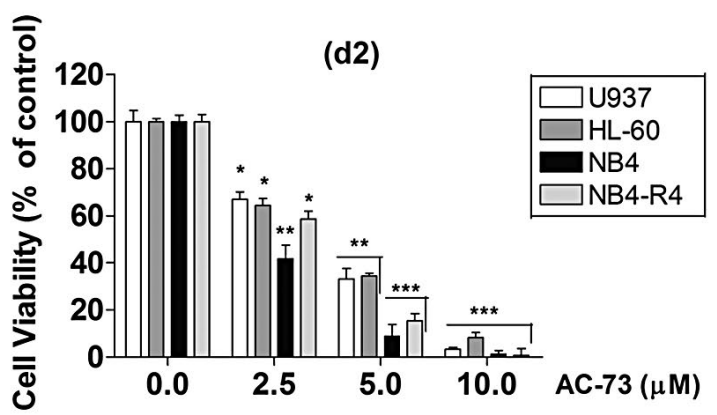

D

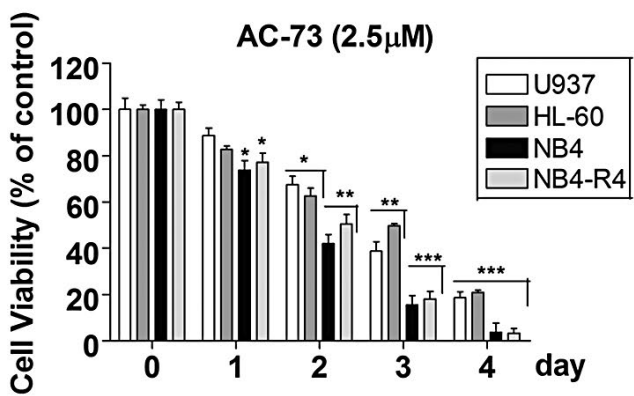

E

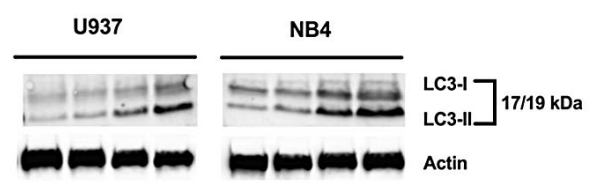

$\begin{array}{lllllllll}- & 2.5 & 5 & 10 & - & 2.5 & 5 & 10 & \text { AC-73 }(\mu \mathrm{M})\end{array}$
$\mathrm{F}$

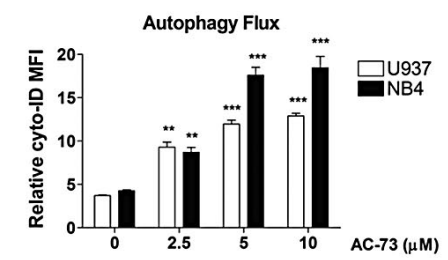

G

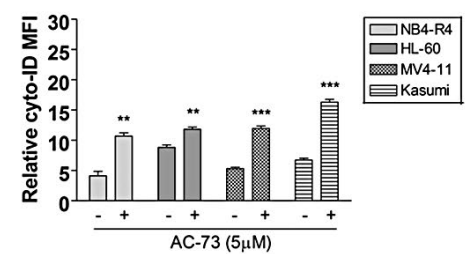

Figure 4. Effects of AC-73 on cell growth, apoptosis and viability of acute myeloid leukemia (AML) cell lines and autophagy. (A) Dose response analysis of AC-73 treatment on leukemic cell growth, as compared to control cells (C). (B) Analysis of the effects of AC-73 treatment performed for 3 days (d3) on leukemic cell apoptosis, as compared to control leukemic cell (0). Total apoptosis by annexin V/PI (\%) detected by using flow cytometric apoptotic assays is indicated. (C and D) Cell viability assays on leukemic cells treated: (C) with AC-73 used at different concentrations for 2 days; (D) at different times with $2.5 \mu \mathrm{M}$ AC-73, as compared to control cells (day 0 ). ( $C$ and D) Viability is presented as percentage viable cell relative to control. (E) Western blot analysis of the autophagy related protein LC 3 and its conversion from LC3-I to LC3-II form in leukemic cells treated with AC-73 used at different concentrations, as compared to control (-) cells. (F) Dose response analysis of AC-73 treatment on the autophagy flux in U937 and NB4 leukemic cells, as compared to control cells (0). (G) Induction of autophagy by AC-73 treatment (+) in several AML cell lines, as compared to control cells (-). (A-D, F and G) Mean \pm Standard Error of Mean is shown. $* P<0.05 ; * * P<0.01 ; * * * P<0.001$. ns: not significant. (E) One representative western blot experiment out of three is shown. Actin was used as an internal control. 
ers previously identified in LSCs. ${ }^{34}$ We confirmed that CD147 is expressed at high levels in all AML subtypes (M1, M2, M3, M5 and M5a) analyzed (Table 1). We also report for the first time that CD147 is co-expressed with CD34, and particularly with CD371, in these cells (Table 1 and Figure 6A). It is interesting to note that in 8 non-M3 AMLs analyzed for CD147 and CD371 expression, there was a strong positive correlation between CD147 and CD371 expression $(P<0.001)$ (Table 1). These data suggest the existence of $\mathrm{CD} 34^{+} / \mathrm{CD} 147^{+}$and $\mathrm{CD} 371^{+} / \mathrm{CD} 147^{+}$subpopulations in AMLs that may have functional properties of leukemic stem cells (LSCs)

Then, we investigated the effects of AC-73 used alone or combined with Ara-C and ATO at low concentration in primary AML blasts, and particularly in M2, M3 and M5 AML cells. We found that AC-73 used alone has no significant effect on AML cell apoptosis, unless used at high concentration $(10 \mu \mathrm{M})$ for $\mathrm{AML}(7)-\mathrm{M} 3$ and $\mathrm{AML}(8)-\mathrm{M} 5$

A
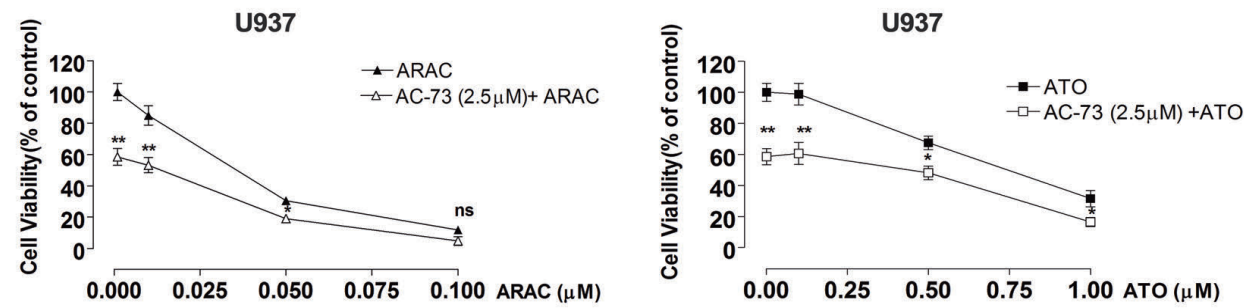

B

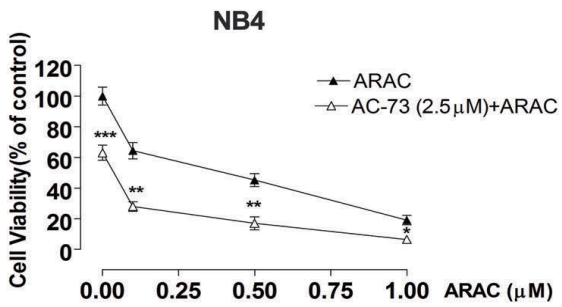

NB4

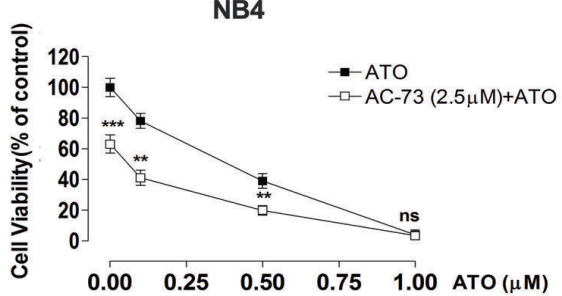

C
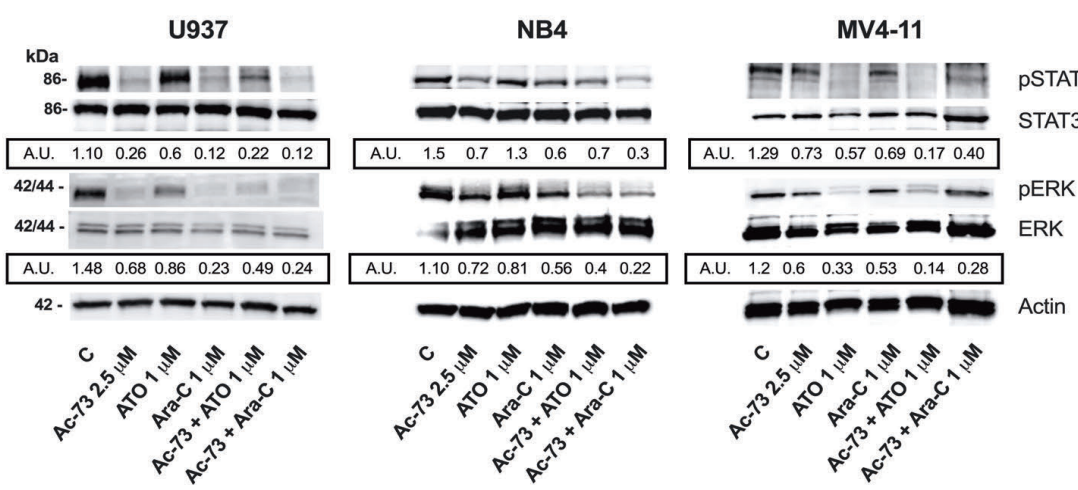

D

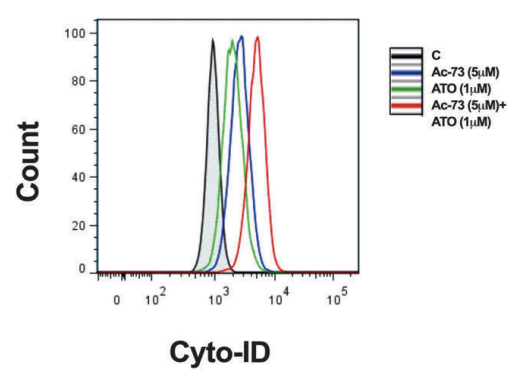

E

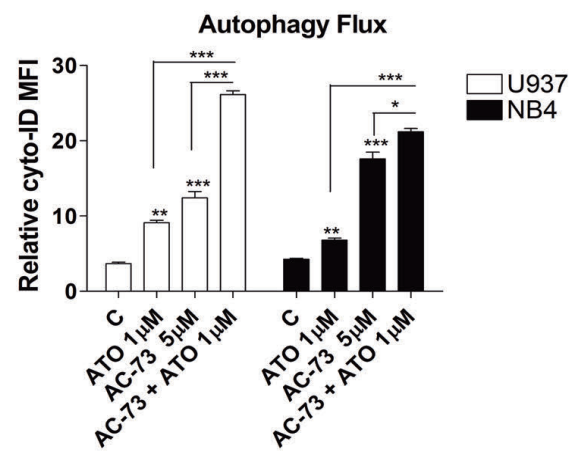

Figure 5. AC-73 potentiates the sensitivity of leukemic cells to chemotherapeutic treatment. (A and $\mathrm{B}$ ) Cell viability assays on U937 and NB4 leukemic cells, treated 24 hours (h) with AC-73 used alone $(2.5 \mu \mathrm{M})$, then in combination with Ara-C (AC-73 + AraC, left panels) or ATO (AC-73 + ATO, right panels) for $48 \mathrm{~h}$, as compared to treatment with single drug, AC-73 (AC-73 + $0.00 \mu \mathrm{M}$ Ara-C; $\mathrm{AC}-73+0.00$ $\mu \mathrm{M}$ ATO), Ara-C and ATO. (C) Western blot analysis of phospho-S TAT 3 ( se r 727 ) (pSTAT3(S727), STAT3, phospho-ERK1/2 (pERK) and ERK1/2 (ERK) protein expression in U937, NB4 and MV4-11 leukemic cell lines. U937, NB4 and MV4-11 cells were treated for 3 days with AC-73 in combination with ATO (AC-73 + ATO 1 $u M)$, or Ara-C (AC-73 + Ara-C 1 $\mu \mathrm{M})$, as compared to AC-73 $(2.5 \mu \mathrm{M})$, ATO $(1 \mu \mathrm{M})$ or Ara-C $(1 \mathrm{uM})$ used alone or to control (C) leukemic cells. Actin was used as an internal control. Quantification of total and phosphorylated ERK1/2 and STAT3 proteins by densitometry analysis is indicated. (D and $E$ ) Analysis of the autophagy flux induces by ATO $(1 \mu \mathrm{M})$ and $\mathrm{AC}-73(5 \mu \mathrm{M})$ used alone or in combination in U937 and NB4 leukemic cells compared to control cells (C). (A, B and E) Mean \pm Standard Error of Mean of three independent experiments is shown. $* P<0.05 ; \quad * * P<0.01$ $* * * P<0.001$. ns: not significant. (C and D) One representative experiment out of three is shown. (C) A.U.: arbitrary units. Ratio pSTAT3/STAT3 and pERK/ERK of three independent experiments is shown. $\mathrm{kDa}$ : molecular weights. 
(Figure 6B). However, AC-73 induces autophagy by increasing the LC3-II/LC3-I ratio in leukemic blasts, as compared to control (-) leukemic cells, even at 2.5 or $5 \mu \mathrm{M}$ (Figure 6C). However, combination of low-dose AC-73 $(2.5 \mu \mathrm{M})$ and ATO or Ara-C $(0.1$ and $1 \mu \mathrm{M})$ more efficiently decreased leukemic blast viability, compared with single AC-73, ATO or Ara treatment (Figure 6D). Overall, our data indicate that AC-73 activates a non-apoptotic autophagic form of cell death in AML blasts and increases the sensitivity of these cells to Ara-C or ATO agents.
Analysis of CD147 expression in acute myeloid leukemia based on TCGA dataset

By providing selected genetic and clinical data from 200 AML patients, useful for prognosis and diagnosis, the TCGA dataset offered a unique opportunity to explore a possible relationship between the recurrent gene mutations observed in AML and the level of CD147 expression. TCGA data set analysis indicates that CD147 level is particularly elevated in AML-M3, those AML subtypes bearing $P M L-R A R A$ fusion gene ${ }^{1,26}$ (Figure 7A, RARA), in
A
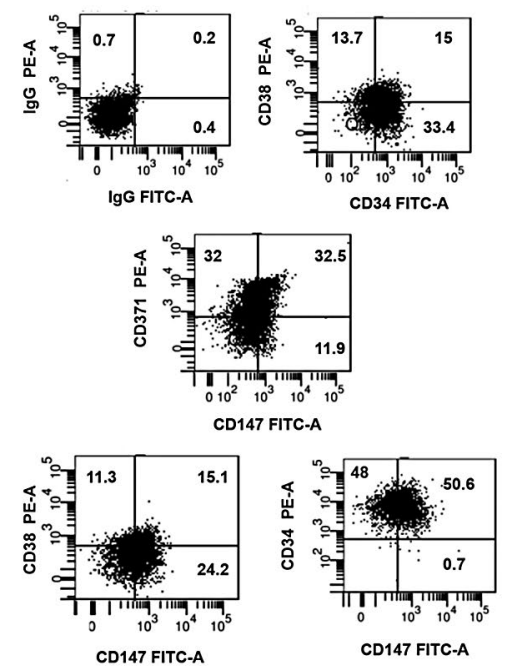

AML (5) M2
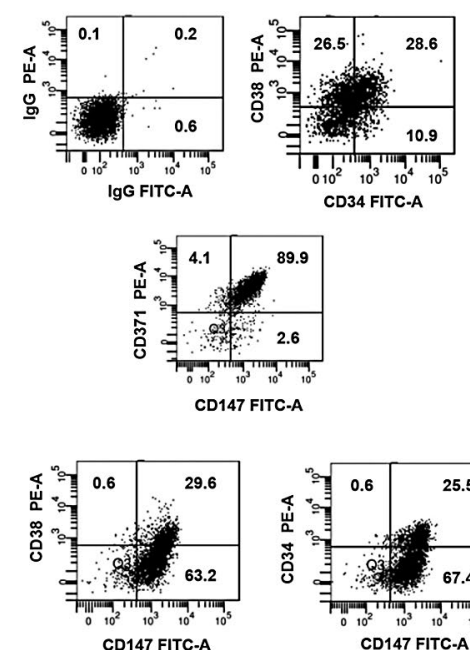

CD147 FITC-A

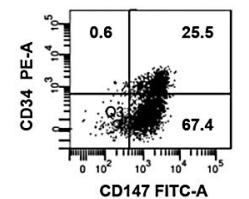

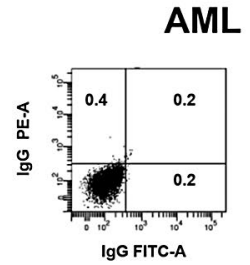

AML (7) M3
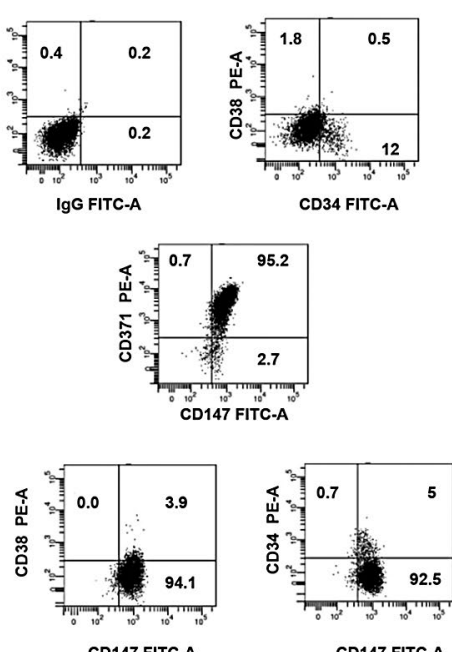

CD147 FITC-A
B

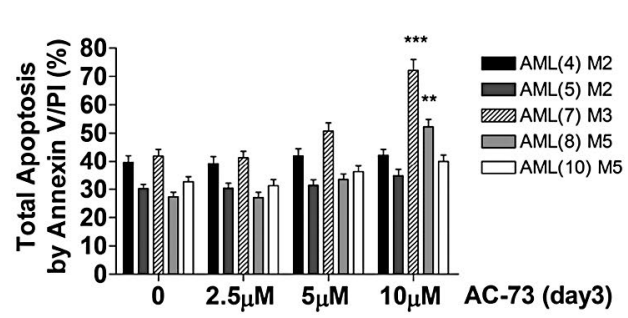

C

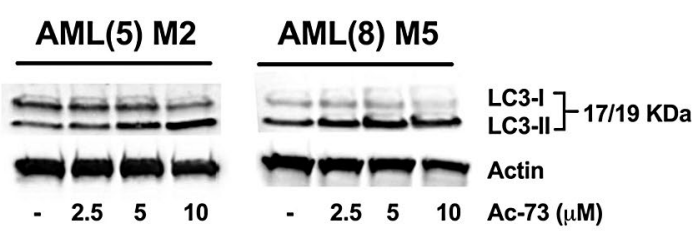

D

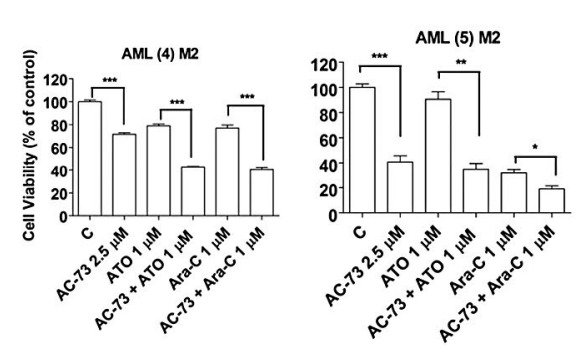

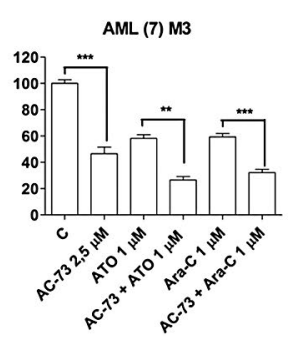
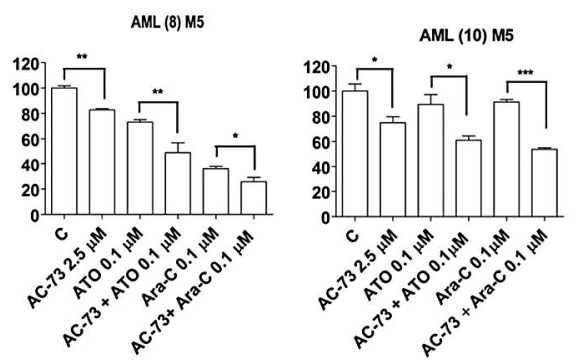

Figure 6. AC-73 decreases the survival of $\mathrm{CD} 147^{+}$acute myeloid leukemia (AML) cells exposed to chemotherapeutic agents and activates autophagy in AML cells. (A) CD147 is expressed in the subfraction of CD34 $\mathrm{CD} 371^{+}$positive AML leukemic cells. (B) Dose response analysis of AC-73 treatment on leukemic cell apoptosis of AML-M2, AML-M3 and AML M5 blasts obtained from 5 AML (4, 5, 7, 8 and 10) patients, as compared to respective control (C) leukemic blasts; flow cytometry analysis of total apoptosis by Annexin V/ PI (\%) is shown. (C) Western blot analysis of the autophagic-related LC3 marker shows its conversion from the LC3-I to LC3-II form in leukemic blasts treated in vitro with growing concentration of AC-73 as compared to control leukemic blasts (-). (D) Cell viability assays were performed on M2, M3 and M5 leukemic blasts, obtained from 5 AML patients (4), (5), (7), (8) and (10), and treated in vitro by AC-73 (2.5 uM) in combination with ATO or Ara-C (0.1 and $1 \mu \mathrm{M}$ ), as compared to control (C) leukemic blasts and to single treatment with AC-73, ATO and Ara-C. (A) One representative experiment is shown. (B and D) Mean \pm Standard Error of Mean of three independent experiments is shown. $* P<0.05$; $* * P<0.01$; $* * * P<0.001$; ns: not significant. (C) One representative western blot experiment out of three is shown. Actin was used as an internal control. 
line with the data observed from fresh samples obtained from AML-M3 patients shown in Figure 2A, while significantly lower levels were observed in AMLs blasts mutated for NPM1, DNMT3A or FLT3 (Figure 7A). By analyzing CD147 levels in all AMLs that we have stratified into three risk groups according to the European LeukemiaNet (ENL) risk classification, we found that: 1) the lower CD147 levels were observed in poor- and intermediaterisk AMLs; 2) surprisingly, the highest CD147 levels were observed in AMLs with favorable risk profile, mainly due to the presence in this group of AML-M3 (Figure 7B). However, it is important to point out that approximately $20 \%$ of poor- and intermediate-risk AMLs also display elevated CD147 expression (Figure 7B). Because AML-M3 have the best prognosis among all AML subtypes for the efficiency of target therapy based on ATRA and ATO, ${ }^{26}$ those patients with favorable risk and high CD147 levels may represent a bias for our analysis. Therefore, we evaluated a possible correlation between CD147 expression level and overall survival of AML patients, excluding AML-M3 patients, and according to CD147 levels classified as: low (CD147<70); medium (CD147; range, 70120); high (CD147 >120) (Figure 7C). In these non-M3 patients who died within 60 months after diagnosis, earlier death (within 20 months) was observed more frequently in cases with high CD147 levels, as compared with (non-M3)-AML subgroups with low-medium CD147 levels ( $P=0.003$ and $P=0.0003$, respectively) (Figure 7D). These three (non-M3)-AML subgroups had comparable age and white blood count (WBC) at diagnosis (Figure 7D, right panels). Our data indicate that, excluding AML-M3, high CD147 levels are correlated with early death of AML patients.

\section{Discussion}

CD147 represents a prognostic marker in several solid tumors and in hematologic malignancies. ${ }^{3,-69,15-19}$ However, the biological function of $\mathrm{CD} 147$ and its potential role as a marker in leukemia remain poorly defined. Apart from the erythrocyte lineage, the expression, regulation and function of CD147 in normal and leukemic hematopoietic cells have not been extensively studied. ${ }^{35}$ CD147, previ- ously identified as a carrier molecule for the blood group antigen $\mathrm{OKa},{ }^{36}$ has been involved in the recirculation of mature erythrocytes from the spleen into the general circulation..$^{37}$ In our study, we show that CD147 is important for the proliferation of normal $\mathrm{CD} 34^{+} \mathrm{HPCs}$ and its expression is down-regulated during Mo and G differentiation. Notably, we found that CD147 is over-expressed in all leukemic cell lines and in the large majority of primary leukemic blasts analyzed, as compared to normal CD34 HPCs. However, CD147 expression significantly decreases during Mo and G differentiation of leukemic cells, thus mimicking what occurs in Mo and G lineage cells. By using AC-73 to inhibit CD147 function in leukemic cell lines and in primary AML blasts, we demonstrated that CD147 overexpression promotes leukemic cell proliferation. Interestingly, we observed that AC-73 treatment inhibits leukemic cell proliferation by suppressing the activation of the ERK/STAT3 signaling pathway, in line with previous studies. ${ }^{5,21}$ As constitutive STAT3 activity has been described to promote AML cell proliferation and survival, ${ }^{22,38}$ targeting key tyrosine kinases upstream of STAT3 has been proposed as a strategy to treat AML. ${ }^{38-40}$ However, due to its role in stem cell renewal, the potential risk of systemic STAT3 inhibition could be a deregulation of hematopoiesis. ${ }^{41}$ Here, we found that CD147 is expressed in normal HPCs and also showed that a low dose of AC-73 induces only a slight inhibition of cell proliferation, without affecting differentiation of $\mathrm{CD}_{4} 4^{+}$ HPCs. Importantly, we found that AC-73 does not cause cell death via apoptosis or cell cycle arrest, but induces autophagy in leukemic cells, as described in previous studies carried out in AML cells treated with ATO as an inducer of autophagy, ${ }^{23}$ or with other novel targeted drugs. ${ }^{23,27,42}$ ${ }^{44}$ In some cases, autophagy accounted for a non-apoptotic decrease in cell viability. ${ }^{22,43}$

To further validate its potential therapeutic activity, we used AC-73 in combination with conventional antileukemia treatment, and showed that the anti-proliferative effect of AC-73 on leukemic cells enhanced the sensitivity of leukemic cells to chemotherapeutic treatments such as Ara-C or ATO, which could be consequently used at lower concentration. Our findings are in line with recent studies showing that other compounds, such as chidamide that down-regulates the JAK2/STAT3 signaling, ${ }^{45}$

Table 1. Analysis of cell surface antigen expression in primary acute myeloid leukemia samples.

\begin{tabular}{lcccccc}
\hline Patient $n$. & FAB subtype & $\begin{array}{c}\text { CD147 } \\
(\%)\end{array}$ & $\begin{array}{c}\text { CD34 } \\
(\%)\end{array}$ & $\begin{array}{c}\text { CD371 } \\
(\%)\end{array}$ & $\begin{array}{c}\text { CD11b } \\
(\%)\end{array}$ & $\begin{array}{c}\text { CD14 } \\
(\%)\end{array}$ \\
\hline 1 & M1 & 63.1 & 21.2 & 80.3 & 31 & 1 \\
2 & M1 & 93.7 & 92.4 & 88.6 & ND & ND \\
\hline 3 & M1 & 44.4 & 98.6 & 64.5 & 28 & 2.3 \\
4 & M2 & 95 & 11.1 & 88.3 & 5 & 1 \\
\hline 5 & M2 & 92.5 & 39.5 & 94 & 4 & 18.5 \\
6 & M3 & 63.7 & 0.2 & 31 & 3.2 & ND \\
\hline 7 & M3 & 97.9 & 12.5 & 95.9 & 60 & 0 \\
\hline 9 & M5 & 71.5 & 5 & 90.9 & 33.6 & 21 \\
10 & M5a & 96.6 & 8.3 & 93.2 & 69 & 8.8 \\
\hline
\end{tabular}

Flow cytometry analysis of CD147,CD34,CD371 and CD11b,CD14 cell surface antigen expression in 10 primary acute myeloid leukemia (AML) samples, obtained from patients with AML pertaining to different French-American-British (FAB) classification subtypes. Results are shown as percentage (\%) of expression. ND: not determined. 
inhibit the viability of AML cells and synergize with cytarabine in inhibiting AML cell viability. ${ }^{46}$ Importantly, we also showed that AC-73 increases ATO-induced autophagy in both $\mathrm{M} 3$ and non-M3 leukemic cells, indicating a possible synergistic anti-tumor interaction of ATO with an inhibitor of CD147, both agents being able to induce autophagy.

The analysis of CD147 expression in AML subsets presented several interesting findings. First, CD147 is overexpressed in all French-American-British classification AML subtypes, and particularly in AML-M3; a finding confirmed through the analysis of primary AML samples. This finding was also corroborated by the analysis of the TCGA data set. Moreover, about 20\% of non-M3 AMLs displayed elevated CD147 levels. Although the overall long-term survival of these patients (defined as highlyexpressing CD147) was similar to the other two non-M3 AML groups (defined as middle- and low-expressing
CD147), death occurs earlier in patients showing high expression of CD147. This observation supports the negative prognostic role of high CD147 expression levels in AMLs, as observed also in other tumors, including some hematologic neoplasia., ${ }^{3,19}$ Other recurrent AML mutations, ${ }^{1,2}$ such as NPM1, FLT3-ITD and DNMT3A, were not associated to particularly elevated levels of CD147. Therefore, AC-73 used in combination with Ara$\mathrm{C}$ or ATO may have clinical potential implications in treatment of AML patients expressing CD147. Future toxicological and pharmacodynamic studies in suitable animal models will be required for a preclinical evaluation of the possible impact of AC-73 as an anti-leukemic drug.

Our study also reports for the first time that CD147 is expressed in a sub-fraction $\mathrm{CD} 34^{+} \mathrm{CD} 371^{+}$of $\mathrm{AML}$ cells that is able to engraft immunodeficient mice, ${ }^{20}$ CD34 being predominantly regarded as a marker of hematopoietic stem cells (HSC) and HPCs, and CD371 as a marker of

\section{AMLs (all subtypes)}

A

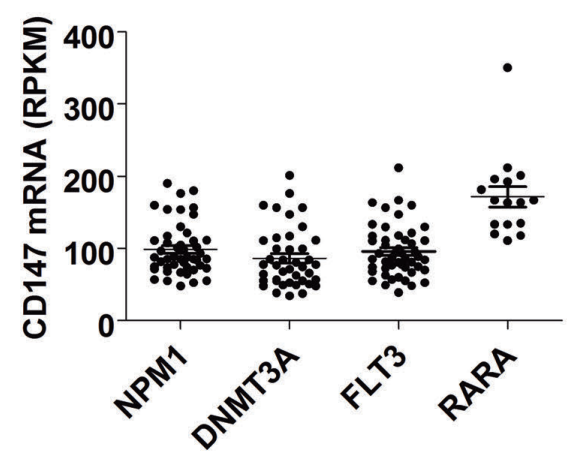

AMLs (without AML-M3)

C

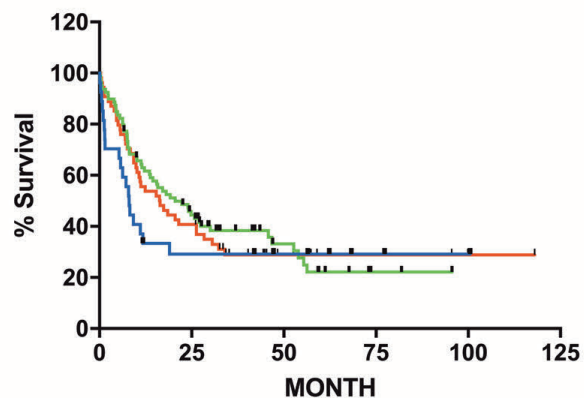

+ low CD147 (<70RPKM)

- medium CD147(70-120RPKM)

+ high CD147(120-350RPKM)
B
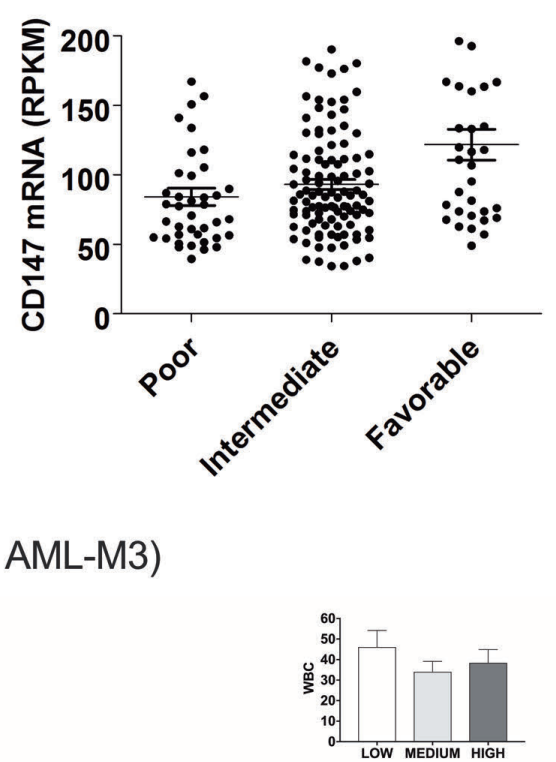

D

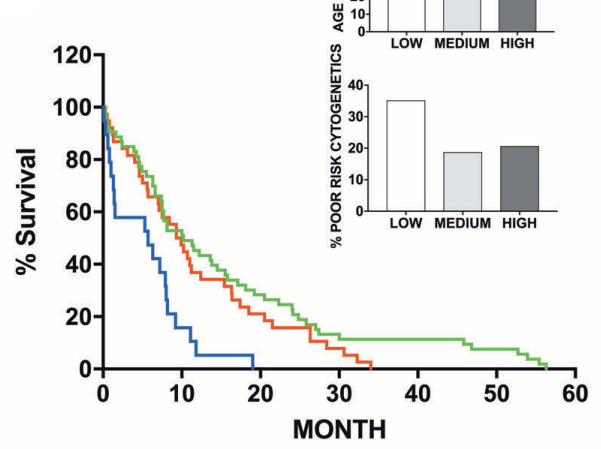

High vs. medium $P=0.0003$

High vs. low $P=0.003$
Figure 7. CD147 gene expression level does not predict overall survival in acute myeloid leukemia (AML), but is correlated in early death of (non-M3)-AML patients. (A) Relationship between the most recurrent gene mutations observed in all AMLs and the leve of CD147 mRNA expression, according to the TCGA dataset. (B) CD147 mRNA levels were analyzed in AMLs stratified into 3 risk groups: poor, intermediate and favorable, according to the European LeukemiaNet (ENL) risk classification. (C) Kaplan-Meier survival analysis in a new group of AML patients that excludes AMLM3 patients, based on CD147 gene expression and stratified according to their CD147 mRNA levels in 3 groups: low (CD147<70); medium (CD147, range, 70-120); high (CD147 $>120$ ). (D) Kaplan-Meier survival analysis in the new group of (nonM3)-AML patients deceased within 50-60 months years after diagnosis, indicates that the kinetics of death is more rapid (within 20 months) among CD147 high patients, as compared with those with low $(P<0.01)$ or medium $(P<0.001)$ CD147 levels; $P$-values calculated by log-rank test. The histograms show that white blood count (WBC) number, age at diagnosis and proportion of patients with poor cytogenetics were comparable in the 3 (non-M3)-AML subgroups subdivided according to CD147 expression level. 
LSCs and a novel prognostic predictor in AML. ${ }^{20,34,47}$ Interestingly, in non-M3 AMLs a strong positive correlation was observed between CD147 and CD371 expression. In this context, while a dedicated future study would shed light on the functional properties of LSCs of the fractions of $\left(\mathrm{CD} 34^{+} \mathrm{CD} 147^{+}\right)$and $\left(\mathrm{CD} 34^{+} \mathrm{CD} 371^{+} \mathrm{CD} 147^{+}\right)$ AML cells, our data suggest that CD147 is expressed in LSCs and may be a potential new prognostic marker in AML and a potential therapeutic target.

Taken together, our study demonstrates a pivotal role for CD147 in leukemic cell proliferation and indicates CD147 as a potential therapeutic target of AC-73 in AML.
Thus, targeting CD147 by using AC-73 or derivate molecules to induce autophagy or to inhibit the growth of leukemic clones, including LSCs, may be an effective option to improve leukemia treatment.

\section{Acknowledgments}

The authors would like to thank AM Cerio for assistance in cell cultures.

\section{Funding}

This study was supported by Istituto Superiore di Sanità (Rome, Italy).

\section{References}

1. Saultz JN, Garzon R. Acute Myeloid Leukemia: A Concise Review. J Clin Med. 2016;5:5(3).

2. Khaled S, Al Malki M, Marcucci G. Acute myeloid leukemia: biologic, prognostic, and therapeutic insights. Oncology (Williston Park). 2016;30(4):318-329.

3. Xiong L, Edwards CK 3rd, Zhou L. The biological function and clinical utilization of CD147 in human diseases: a review of the current scientific literature. Int J Mol Sci. 2014;15(10):17411-17441.

4. Somno A, Anuchapreeda S, Chruewkamlow N, Pata S, Kasinrerk W, Chiampanichayakul S. Involvement of CD147 on multidrug resistance through the regulation of P-glycoprotein expression in K562/ADR leukemic cell line. Leuk Res Rep. 2016;6:33-38.

5. Bai Y, Huang W, Ma LT, Jiang JL, Chen ZN. Importance of $\mathrm{N}$-glycosylation on CD147 for its biological functions. Int J Mol Sci. 2014;15(4):6356-6377.

6. Grass GD, Dai L, Oin Z, Parsons C, Toole BP. CD147: regulator of hyaluronan signaling in invasiveness and chemoresistance. Adv Cancer Res. 2014;123:351-373.

7. Tang J, Guo YS, Zhang Y, et al. CD147 induces UPR to inhibit apoptosis and chemosensitivity by increasing the transcription of Bip in hepatocellular carcinoma. Cell Death Differ. 2012;19(11):1779-1790.

8. Gao H, Jiang Q, Han Y, Peng J, Wang C. shRNA-mediated EMMPRIN silencing inhibits human leukemic monocyte lymphoma U937 cell proliferation and increases chemosensitivity to adriamycin. Cell Biochem Biophys. 2015;71(2):827-835.

9. Huang Z, Wang L, Wang Y, et al. Overexpression of CD147 contributes to the chemoresistance of head and neck squamous cell carcinoma cells. J Oral Pathol Med. 2013;42(7):541-546.

10. Muramatsu T. Basigin (CD147), a multifunctional transmembrane glycoprotein with various binding partners. J Biochem. 2016;159(5):481-490.

11. Le Floch R, Chiche J, Marchiq I, et al. CD147 subunit of lactate/H+ symporters MCT1 and hypoxia-inducible MCT4 is critical for energetics and growth of glycolytic tumors. Proc Natl Acad Sci U S A. 2011;108(40):16663-16668.

12. Kirk P, Wilson MC, Heddle C, Brown MH, Barclay AN, Halestrap AP. CD147 is tightly associated with lactate transporters MCT1 and MCT4, and facilitates their cell surface expression. EMBO J. 2000;19(15):38963904.

13. Eliasson P, Jönsson JI. The hematopoietic stem cell niche: low in oxygen but a nice place to be. J Cell Physiol. 2010;222(1):17 22

14. Testa U, Labbaye C, Castelli G, Pelosi E Oxidative stress and hypoxia in norma and leukemic stem cells. Exp Hematol. 2016;44(7):540-560.

15. Fu J, Fu J, Chen X, Zhang Y, Gu H, Bai Y CD147 and VEGF co-expression predicts prognosis in patients with acute myeloid leukemia. Jpn J Clin Oncol. 2010;40 (11):1046-1052.

16. Honnemyr M, Bruserud $\varnothing$, Brenner AK The constitutive protease release by primary human acute myeloid leukemia cells J Cancer Res Clin Oncol. 2017; 143(10):1985-1998.

17. Zhu D, Wang Z, Zhao JJ, et al. The Cyclophilin A-CD147 complex promotes the proliferation and homing of multiple myeloma cells. Nat Med. 2015;21(6):572580.

18. Eichner R, Heider M, Fernández-Sáiz V, et al. Immunomodulatory drugs disrupt the cereblon-CD147-MCT1 axis to exert antitumor activity and teratogenicity. Nat Med. 2016;22(7):735-743.

19. Panchabhai S, Schlam I, Sebastian S, Fonseca R. PKM2 and other key regulators of Warburg effect positively correlate with CD147 (EMMPRIN) gene expression and predict survival in multiple myeloma. Leukemia. 2017;31(4):991-994.

20. van Rhenen A, van Dongen GA, Kelder A et al. The novel AML stem cell associated antigen CLL-1 aids in discrimination between normal and leukemic stem cells. Blood. 2007;110(7):2659-2666.

21. Fu ZG, Wang L, Cui HY, et al. A novel small-molecule compound targeting CD147 inhibits the motility and invasion of hepatocellular carcinoma cells. Oncotarget. 2016;7(8):9429-9447.

22. Redell MS, Ruiz MJ, Alonzo TA, Gerbing $\mathrm{RB}$, Tweardy DJ. Stat3 signaling in acute myeloid leukemia: ligand-dependent and independent activation and induction of apoptosis by a novel small-molecule Stat3 inhibitor. Blood. 2011;117(21):5701-5709.

23. Orsini M, Morceau F, Dicato M, Diederich M. Autophagy as a pharmacological target in hematopoiesis and hematological disorders. Biochem Pharmacol. 2018;152:347 361.
24. Folkerts H, Hilgendorf S, Wierenga ATJ, et al. Inhibition of autophagy as a treatment strategy for p53 wild-type acute myeloid leukemia. Cell Death Dis. 2017;8(7):e2927.

25. Jin J, Britschgi A, Schläfli AM, et al. Low Autophagy (ATG) Gene Expression Is Associated with an Immature AML Blast Cell Phenotype and Can Be Restored during AML Differentiation Therapy. Oxid Med Cell Longev. 2018;2018:1482795.

26. Lo-Coco F, Cicconi L, Breccia M. Current standard treatment of adult acute promyelocytic leukaemia. Br J Haematol. 2016;172(6):841-854.

27. Goussetis DJ, Altman JK, Glaser H, McNeer JL, Tallman MS, Platanias LC. Autophagy is a critical mechanism for the induction of the antileukemic effects of arsenic trioxide. J Biol Chem. 2010; 285(39):29989-29997.

28. Lulli V, Romania P, Riccioni $\mathrm{R}$, et al. Transcriptional silencing of the ETS1 oncogene contributes to human granulocytic differentiation. Haematologica. 2010; 95(10):1633-1641.

29. Spinello I, Quaranta MT, Paolillo R, et al Differential hypoxic regulation of the microRNA-146a/CXCR4 pathway in normal and leukemic monocytic cells: impact on response to chemotherapy. Haematologica. 2015;100(9):1160-1171.

30. Labbaye C, Spinello I, Quaranta MT, et al. A three-step pathway comprising $\mathrm{PLZF/miR-146a/CXCR4} \mathrm{controls}$ megakaryopoiesis. Nat Cell Biol. 2008;10(7):788-801

31. Tanida I, Ueno T, Kominami E. LC3 and Autophagy. Methods Mol Biol. 2008; 445:77-88.

32. Benekli M, Xia Z, Donohue KA, et al. Constitutive activity of signal transducer and activator of transcription 3 protein in acute myeloid leukemia blasts is associated with short disease-free survival. Blood. 2002;99(1):252-257.

33. Lunghi P, Tabilio A, Dall'Aglio PP, et al. Downmodulation of ERK activity inhibits the proliferation and induces the apoptosis of primary acute myelogenous leukemia blasts. Leukemia. 2003;17(9):1783-1793.

34. Pelosi E, Castelli G, Testa U. Targeting LSCs through membrane antigens selectively or preferentially expressed on these cells. Blood Cells Mol Dis. 2015;55(4):336346.

35. Papayannopoulou $\mathrm{T}$, Brice $\mathrm{M}$. Integrin expression profiles during erythroid differentiation. Blood. 1992;79(7):1686-1694. 
36. Spring FA, Holmes CH, Simpson KL, et al. The Oka blood group antigen is a marker for the M6 leukocyte activation antigen, the human homolog of OX-47 antigen, basigin and neurothelin, an immunoglobulin superfamily molecule that is widely expressed in human cells and tissues. Eur J Immunol. 1997;27(4):891-897.

37. Coste I, Gauchat JF, Wilson A, et al. Unavailability of CD147 leads to selective erythrocyte trapping in the spleen. Blood. 2001;97(12):3984-3988.

38. Xiong A, Yang Z, Shen Y, Zhou J, Shen $Q$. Transcription Factor STAT3 as a Novel Molecular Target for Cancer Prevention. Cancers (Basel). 2014;6(2):926-957.

39. Murone M, Radpour R, Attinger A, et al. The Multi-kinase Inhibitor Debio 0617B Reduces Maintenance and Self-renewal of Primary Human AML CD34+
Stem/Progenitor Cells. Mol Cancer Ther 2017;16(8):1497-1510.

40. Xiang M, Kim H, Ho VT, et al. Gene expression-based discovery of atovaquone as a STAT3 inhibitor and anticancer agent. Blood. 2016;128(14):1845-1853.

41. Yu H, Pardoll D, Jove R. STATs in cancer inflammation and immunity: a leading role for STAT3. Nat Rev Cancer. 2009; 9(11):798-809.

42. Evangelisti C, Evangelisti C, Chiarini F, et al. Autophagy in acute leukemias: a double-edged sword with important therapeutic implications. Biochim Biophys Acta. 2015;1853(1):14-26.

43. Wei Y, Kadia T, Tong W, et al. The combination of a histone deacetylase inhibito with the Bcl-2 homology domain-3 mimetic GX15-070 has synergistic antileukemia activity by activating both apoptosis and autophagy. Clin Cancer Res. 2010 16(15):3923-3932.

44. Auberger P, Puissant A. Autophagy, a key mechanism of oncogenesis and resistance in leukemia. Blood. 2017;129(5):547-552.

45. Zhao S, Guo J, Zhao Y, et al. Chidamide, a novel histone deacetylase inhibitor, inhibits the viability of MDS and AML cells by suppressing JAK2/STAT3 signaling. Am J Transl Res. 2016;8(7):3169-3178.

46. Li X, Yan X, Guo W, et al. Chidamide in FLT3-ITD positive acute myeloid leukemia and the synergistic effect in combination with cytarabine. Biomed Pharmacother 2017;90:699-704.

47. Wang YY, Chen WL, Weng XO, et al. Low CLL-1 Expression Is a Novel Adverse Predictor in 123 Patients with De Novo CD34+ Acute Myeloid Leukemia. Stem Cells Dev. 2017;26(20):1460-1467. 